\title{
SCIENZA E MATEMATICA: IL CASO DELL'INDISPENSABILITÀ
}

\author{
ENRICO CINTI (*)
}

\begin{abstract}
SunTO. - In questo breve contributo, introdurrò una delle più note e discusse conseguenze dell'interazione tra matematica e scienza, l'argomento per l'indispensabilità delle entità matematiche di Putnam e Quine. Partiremo dal vederne la formulazione standard, e come esso sia particolarmente stringente per i realisti scientifici. In seguito, andremo ad analizzare le componenti principali dell'argomento: l'indispensabilità, il naturalismo e l'olismo della conferma. Nello specifico, cercherò di mostrare anche come naturalismo e olismo diano origine allo specifico tipo di realismo scientifico che sta alla base dell'argomento di Putnam e Quine. Infine, esporrò brevemente alcune delle obiezioni e delle questioni irrisolte legate all'argomento.
\end{abstract}

$* * *$

ABSTRACT. - In this brief contribution, I will introduce one of the most famous consequences of the interaction between science and mathematics, Putnam and Quine's argument for the indispensability of mathematical entities. We will start by looking at its standard formulation, and how it is particularly cogent for scientific realists. After this, we will look at the main components of the argument, that is indispensability, naturalism and confirmational holism. Furthermore, we will see how naturalism and confirmational holism give rise to the specific type of scientific realism that underlies Putnam and Quine's argument. Finally, we will look at some objections and unresolved issues connected to the argument.

In questo contributo è mia intenzione introdurre e illustrare alcune interessanti questioni legate a quello che è uno dei più discussi $\mathrm{e}$ celebri argomenti nella filosofia della matematica del XX secolo, l'argomento per l'indispensabilità delle entità matematiche di Putnam e Quine.

(*) Università degli Studi di Milano, Italia. E-mail: enrico.cinti@studenti.unimi.it 
L'argomento, volto a difendere il realismo rispetto alle entità matematiche, altresì detto Platonismo, parte dalla constatazione della necessità dell'uso della matematica nella teorizzazione scientifica. Da questo deriva come conseguenza l'obbligo, da parte del realista scientifico, ovverosia colui che pensa che le teorie scientifiche siano descrizioni approssimativamente vere del mondo, di riconoscere nell'insieme delle entità nei confronti delle quali è impegnato ontologicamente le entità matematiche, ovvero le entità, quali numeri, insiemi e funzioni, che fanno parte delle discipline matematiche. Con essere impegnati ontologicamente verso una certa entità si intende riconoscere l'esistenza di quell'entità.

Il contributo è diviso in sezioni. Nella prima presenterò l'argomento di Putnam e Quine e traccerò una minima parte della sua preistoria filosofica, e spiegherò perché l'argomento sia particolarmente stringente per i realisti scientifici. Nella seconda andrò ad analizzare le premesse dell'argomento, e nello specifico vedrò cosa si intenda per indispensabilità nella seconda premessa e quindi le due presupposizioni fondamentali della prima premessa di questo argomento, quella del naturalismo e quella dell'olismo. Nella terza invece vedrò brevemente alcune obiezioni classiche all'argomento di Putnam e Quine, e quindi mi avvierò ad una breve conclusione.

\section{L'argomento di Putnam e Quine}

L'argomento di Putnam e Quine per l'indispensabilità delle entità matematiche risale alla seconda metà del XX secolo, e pone le sue radici nella tradizione metafilosofica sviluppata da Quine dagli anni '30 in avanti, allo scopo di riabilitare la metafisica e l'ontologia dopo le critiche dei positivisti logici e darle una fondazione più rigorosamente scientifica. L'elemento cardine di questa visione del metodo filosofico è l'idea di impegno ontologico, ovvero il metodo tramite cui si stabiliscono le entità che vengono considerate come esistenti. Queste entità, per Quine, vanno individuate tramite la traduzione in logica del primo ordine delle migliori teorie scientifiche disponibili. Fatto questo, si procede a estrarre l'impegno ontologico delle teorie in questione andando a leggere cosa sia contenuto nel dominio dei quantificatori presenti nella parafrasi precedentemente fatta. Le entità così individuate sono quelle nei confronti delle quali dobbiamo essere impegnati ontologica- 
mente, se crediamo nella teoria in questione. In questa maniera, la metafisica smette di essere mera astrazione, e viene fermamente ancorata e resa continua con il lavoro delle scienze empiriche.

Tutto questo è importante per l'argomento per l'indispensabilità delle entità matematiche perché la conclusione dell'argomento, ovvero la necessità di impegnarsi ontologicamente verso le entità matematiche, segue dalla necessità dell'uso della matematica nelle teorie scientifiche. In questo senso, rappresenta una perfetta istanza dell'approccio quineano all'ontologia.

Arrivati a questo punto, è utile esporre l'argomento in maniera precisa, prima di procedere ulteriormente nella discussione.

L'argomento per l'indispensabilità delle entità matematiche di Putnam e Quine è così articolato:

- Dobbiamo essere impegnati ontologicamente verso tutte e sole le entità indispensabili per le mostre migliori teorie scientifiche (Premessa 1).

- Le entità matematiche sono indispensabili per le mostre migliori teorie scientifiche (Premessa 2).

- Dobbiamo essere impegnati ontologicamente verso le entità matematiche (Conclusione)

L'argomento esposto sopra è valido. Di conseguenza, chiunque desideri rifiutarne la conclusione, dovrà motivare il rifiuto di almeno una delle premesse. Più avanti le analizzeremo nel dettaglio, e vedremo inoltre alcuni tentativi in questo senso.

Ora però, per concludere questa prima sezione, illustrerò brevemente perché l'argomento sia particolarmente stringente per i realisti scientifici.

Questo è dovuto al fatto che l'argomento sopra esposto è un caso di abduzione, anche detta inferenza alla miglior spiegazione. L'abduzione è una forma di inferenza tipicamente accettata dai realisti scientifici, e anzi argomenti di tipo abduttivo costituiscono una delle basi su cui vengono costruiti e difesi molti argomenti a favore del realismo scientifico, quale ad esempio è il cosiddetto "argomento niente miracoli” (Putnam 1975). Non solo, ma la forma specifica delle premesse e della conclusione dell'argomento di Putnam e Quine è fondamen- 
talmente simile a quella degli argomenti normalmente usati dai realisti scientifici per motivare la loro accettazione delle entità teoriche delle teorie scientifiche.

In questo senso, un nominalista che desiderasse essere anche realista scientifico si troverebbe nella situazione di accettare un ragionamento abduttivo per motivare il suo realismo e al contempo doverne rifiutare uno estremamente simile per non trovarsi a dover ammettere l'esistenza delle entità matematiche. Questo è quello che Quine (1980) chiama il "doppio standard dell'ontologia".

\section{INDISPENSABILITÀ, NATURALISMO E OLISMO: LE PREMESSE DELl'argomento di Putnam E Quine}

Vista la struttura dell'argomento e chiarita la sua forza per i realisti scientifici, possiamo ora passare in questa sezione ad analizzare più nel dettaglio le premesse dell'argomento e alcune delle presupposizioni che vi fanno da sfondo.

Cominceremo quest'analisi andando a vedere più nel dettaglio che cosa si intenda per indispensabilità quando vi si fa riferimento nella seconda premessa dell'argomento, e inoltre vedremo quanto della matematica l'indispensabilità qui discussa possa effettivamente giustificare ontologicamente.

È importante chiarire che in questo caso indispensabilità non significa semplicemente non eliminabilità, ma che oltre a ciò la teoria risultante sia più attraente. È utile spiegare cosa si intenda con questo.

Rifacendoci alla breve discussione fatta prima a riguardo della metafilosofia di Quine, è utile notare che le parafrasi lì discusse non sono necessariamente uniche, nel senso che vi potrebbero essere molteplici parafrasi in logica del primo ordine della stessa teoria scientifica. Ogni parafrasi può generare una diversa ontologia, e in casi del genere, per determinare quale sia da preferirsi, ci si affida agli stessi criteri tipici della buona teorizzazione scientifica: semplicità, eleganza, potere esplicativo, adeguatezza empirica e fertilità, per citarne alcuni. Questi stessi criteri sono alla base del senso di indispensabilità utilizzato in questo argomento: un'entità è indispensabile a una teoria scientifica se la teoria che ne risultasse espungendola fosse peggiore secondo i criteri sopra esposti, un criterio che vale per le entità matematiche così come vale per qualunque altra entità, e che stringe ulteriormente 
il legame, nella nostra prospettiva, tra ontologia, scienza, matematica e indispensabilità.

Un'ulteriore questione interessante è quanto della matematica l'argomento di Putnam e Quine effettivamente giustifichi. Questo è dovuto al fatto che l'argomento si occupa esplicitamente della parte della matematica indispensabile alle teorie scientifiche. Ma questo non esaurisce tutto l'universo della matematica. Sia Quine che Putnam sono consapevoli di questo fatto (Putnam 1979, Quine 1986), e hanno risposte di vario genere al problema. In generale, sembrano accettare che il loro argomento non giustifichi l'intero della matematica ma solo quelle parti che trovano applicazione nelle scienze empiriche. Nonostante ciò, vengono comunque accettate alcune parti della matematica anche se prive di applicazione diretta, in virtù del fatto che sono conseguenza di parti che hanno applicazione, e di conseguenza ereditano così la loro giustificazione. Questo comunque non permette di estendere l'argomento all'intero della matematica, ma permette comunque di allargarne il campo d'azione.

Passiamo ora a considerare le due principali presupposizioni che fanno da sfondo alla prima premessa dell'argomento, ovvero il naturalismo e l'olismo.

Nello specifico, vedremo che il naturalismo ci fornirà ragione per avere impegno ontologico solo nelle entità postulate dalle nostre migliori teorie scientifiche, mentre l'olismo ci darà ragione per avere impegno ontologico verso tutte queste entità.

Il naturalismo di Quine può essere riassunto nell'idea che non esista nessuna "filosofia prima", per citare Aristotele, e che l'impresa filosofica va condotta in maniera continua con l'impresa scientifica (Quine 1981). In questo senso, dunque, la filosofia è vista come parte e continuazione della scienza, e non viene considerata come una disciplina che da una posizione privilegiata guarda sul mondo e valuta e decide cosa sia valido e cosa no della nostra conoscenza, come un giudice. Piuttosto, diventa collaboratrice delle scienze empiriche nella determinazione di tutto quello che c'è da sapere sul mondo e nel fornirci conoscenza su di esso, come pari, senza nessun tipo di privilegio.

È Quine stesso a collegare questa dottrina col realismo scientifico, indicandone l'origine nel "ostinato realismo, la solida convinzione dello scienziato naturale che non ha mai provato alcun dubbio al di là delle discutibili incertezze interne alla scienza" ("unregenerate realism, the robust state of mind of the natural scientist who has never felt any 
qualms beyond the negotiable uncertainties internal to science" (Quine 1981) traduzione dell'autore). In questo senso, dunque, la dottrina del naturalismo nasce dal riconoscimento degli incredibili successi ottenuti dalle scienze empiriche nel fornirci conoscenza affidabile sul mondo che abitiamo, e dell'efficacia del metodo scientifico come metodo per acquisire informazioni vere sulla realtà.

Il naturalista si impegna in conseguenza di ciò a ricercare i propri impegni ontologici e a costruire le proprie teorie metafisiche sulla base delle informazioni ricavate dalle teorie scientifiche, e a non tenere conto di informazioni ottenute attraverso metodi diversi da quello scientifico, per quanto convinto egli possa essere della loro verità. Egli cerca quindi nella sua pratica filosofica di riprodurre il più possibile la buona pratica scientifica e di mutuarne i metodi, utilizzandone i risultati come punti di partenza per il suo stesso lavoro.

La dottrina del naturalismo, dunque, ci giustifica nel nostro guardare soltanto alle migliori teorie scientifiche disponibili per determinare i nostri impegni ontologici, e quindi le entità che si è disposti ad ammettere come esistenti. Per arrivare a giustificare la prima premessa, tuttavia, manca ancora un ulteriore passo. Abbiamo infatti così ottenuto ragione per credere nell'esistenza delle sole entità richieste dalle nostre migliori teorie scientifiche. Dobbiamo ancora giustificare il credere nell'esistenza di tutte le entità richieste dalle nostre migliori teorie scientifiche. Si potrebbe infatti pensare, e sarebbe compatibile con il naturalismo esposto sopra, che alcune delle entità richieste dalle nostre teorie non vadano comunque ammesse nel novero di quelle contate come esistenti (per esempio quelle matematiche, come vorrebbe il nominalista). Quindi, per completare la giustificazione della prima premessa, e dunque dell'argomento, dobbiamo ricorrere a un'ulteriore dottrina, l'olismo della conferma, che ci dice che se una teoria è confermata, lo è come un intero, e non solo in alcune sue parti mentre in altre no.

L'olismo della conferma, a volte chiamato anche tesi di QuineDuhem (Quine 1980), è la tesi per cui le teorie vengono confermate come interi, come blocchi unici, e quindi che la fiducia che le scoperte empiriche ci danno vale per qualunque aspetto e elemento della teoria, non solo per alcune sue parti. Questo è dovuto al fatto che, nel momento in cui si esegue un esperimento per testare una teoria, il risultato ottenuto è dovuto a qualunque elemento coinvolto nella costruzione e nel test della teoria, non solo a quello che viene contato come strettamente parte della teoria. Per esempio, se si esegue un esperimento per verificare o meno se sia 
vero che tutti i metalli si espandono quando scaldati, scaldando un pezzo di rame, e si osserva che il pezzo di rame non si espande, questo può essere dovuto sia al fatto che la nostra teoria per cui tutti i metalli si espandono quando scaldati è falsa, ma anche per esempio a un errore nella costruzione del macchinario usato nell'esperimento, o nelle pratiche ingegneristiche che usiamo, $\mathrm{o}$ anche al fatto che $2+2=4$ sia falso. Tutte queste sono ipotesi che sono in grado di spiegare il risultato sperimentale, e senza implicare nulla sulla verità o falsità della specifica teoria che viene testata.

Senza addentrarci troppo in questioni di filosofia della scienza, possiamo dire che da questo segue che, nel momento in cui si accumuli evidenza positiva per una teoria, la si accumula per la teoria sommata a tutte le altre teorie ed ipotesi che le fanno da sfondo e che permettono, tra le altre cose, di eseguire esperimenti e derivare predizioni. Tra queste ipotesi e teorie va contata anche la matematica utilizzata nella specifica teoria sotto esame.

Nello specifico del nostro caso, questo significa che la stessa evidenza empirica che viene utilizzata per giustificare la credenza in qualunque aspetto materiale, osservabile o no, della teoria, viene usata anche per giustificare tra le altre cose credenza in tutta la matematica che vi è utilizzata.

In questo senso, dunque, la matematica utilizzata in una teoria riceve una giustificazione che è esattamente la stessa che ricevono le parti concrete ed empiriche di quella teoria, avendo questa giustificazione la stessa origine e contenuto, ovvero gli esperimenti eseguiti dagli scienziati per testare una teoria.

Detto questo, dunque, possiamo notare che grazie all'olismo della conferma abbiamo la condizione che ci mancava per giustificare la prima premessa, e cioè che dobbiamo impegnarci verso tutte le entità necessarie a una teoria scientifica. Unendo naturalismo e olismo della conferma, dunque, possiamo giustificare completamente la prima premessa.

\section{ALCUNE OBIEZIONI}

A questo punto, avendo finito di analizzare più nel dettaglio la giustificazione per le premesse dell'argomento per l'indispensabilità delle entità matematiche di Putnam e Quine, possiamo passare a vedere brevemente alcune obiezioni all'argomento, nel tentativo di difendere la possibilità di conciliare realismo scientifico e nominalismo. 
Una prima obiezione, molto naturale, all'argomento è che, per quanto riesca effettivamente a dare una motivazione per l'impegno ontologico nei confronti delle entità matematiche, l'argomento non offre nessun tipo di spiegazione sul perché effettivamente la matematica sia così utile per le scienze (si veda per esempio Kitcher 1984). In un certo senso, si può vedere questa come un'obiezione che l'argomento, pur essendo valido, manca di cogliere il punto di quella che è "l'irragionevole efficacia della matematica nelle scienze naturali", come titolava un classico articolo di Wigner (Wigner 1960).

Un'altra importante obiezione, che ha dato origine a uno dei più importanti programmi di ricerca nell'ontologia della matematica, e che ha dominato molte discussioni in filosofia della matematica dagli anni '80 in poi, è il tentativo di Hartry Field di nominalizzare le scienze naturali (Field 1980).

Il punto della critica di Field è di dimostrare che la seconda premessa, che afferma l'indispensabilità della matematica alle nostre teorie scientifiche, non è in ultima istanza vera, e quindi di rigettare l'argomento su queste basi.

Dopo aver difeso l'idea che il motivo per cui la matematica è particolarmente utile nelle scienze empiriche risiede nel fatto che permettono una formulazione particolarmente chiara e utile a derivare predizioni delle teorie stesse, Field si dedica a sviluppare quello che è il risultato più significativo del suo programma di ricerca, ovvero la dimostrazione che le teorie scientifiche possono essere espresse senza fare uso della matemati$\mathrm{ca}$, senza per questo doverci trovare ad avere a che fare con formulazioni molto poco attraenti, nel senso di cui abbiamo discusso nella precedente sezione parlando del significato di indispensabilità, delle teorie stesse.

A questo scopo, Field riformula un frammento della teoria della gravitazione di Newton in termini di punti dello spaziotempo e relazioni di contiguità tra questi, senza fare il minimo riferimento ad alcuna entità matematica. Ovviamente questo non equivale ad una dimostrazione che una procedura del genere può essere eseguita per ogni teoria, ma quello che i sostenitori di questo programma vogliono difendere è che essendo stato dimostrato che la matematica può essere eliminata da una teoria scientifica "tipica", nel senso di poter fare in molti suoi aspetti da prototipo per come una teoria scientifica è formulata, questo renda plausibile pensare che una simile procedura possa essere estesa a qualunque altra teoria, sebbene questo non sia stato mostrato esplicitamente.

Il progetto di Field di nominalizzare le teorie scientifiche è stato 
ampiamente discusso e criticato, specialmente riguardo alla possibilità di estendere effettivamente la sua costruzione di una teoria nominalistica a tutte le altre teorie scientifiche di interesse. Nello specifico, una della critiche più stringenti in questo senso è quella dovuta a Malament (1982), che mette in dubbio la possibilità di estendere il processo di nominalizzazione di Field al di là delle teorie di campo classiche. Questo perché il metodo sviluppato da Field è un metodo per riformulare una teoria espressa in termini di proprietà assegnate a punti di un certo spazio in termini esclusivamente dei punti stessi e di alcune relazioni tra essi. Fintanto che, come nel caso delle teorie di campo classiche, lo spazio in questione è lo spaziotempo, la riformulazione risultante sarà in termini esclusivamente nominalistici, essendone stato espunto qualunque riferimento a entità matematiche, sostituito con riferimenti ai punti dello spaziotempo e alle loro mutue relazioni. È con questa metodologia che Field riformula la teoria della gravitazione di Newton.

Malament fa notare che la condizione per cui lo spazio su cui è formulata la teoria e lo spaziotempo fisico coincidono è necessaria al programma di Field, ma fa poi notare che questa condizione non è soddisfatta da un gran numero di teorie, in buona sostanza dalla gran parte delle teorie che non sono teorie classiche di campo.

Più nel dettaglio, Malament fa riferimento alle teorie classiche hamiltoniane e alla meccanica quantistica. L'aspetto rilevante per i nostri scopi delle teorie hamiltoniane è che sono costruite tramite il cosiddetto spazio delle fasi, ovvero uno spazio matematico ogni punto del quale rappresenta un possibile stato del sistema in questione. Quello che ci interessa è che questo spazio non è lo spaziotempo fisico, essendo piuttosto lo spazio dei possibili stati di un certo sistema. Di conseguenza, il massimo che il metodo di Field può offrire per teorie del genere è una riformulazione in termini di stati possibili e relazioni tra questi. Ma ovviamente questa riformulazione non ha nulla di necessariamente nominalistico, e di conseguenza non garantisce a Field il risultato sperato di espungere la matematica dalle scienze naturali.

Il caso diventa ancora più complesso quando ci si sposta a considerare la meccanica quantistica, che è formulata tramite i cosiddetti spazi di Hilbert, anch'essi spazi ogni elemento dei quali rappresenta un possibile stato, ma che oltre a questo presentano ulteriori complicazioni matematiche che li rendono ancora più ostili al progetto nominalista di Field. Malament in questo modo mostra dunque come l'idea che l'esempio della gravitazione di Newton possa fare da archetipo per tutte le altre teo- 
rie scientifiche si regga su terreni molto fragili, per non dire frananti.

Nonostante ciò, si è tentato da parte di vari nominalisti di mostrare come il programma di Field possa essere esteso anche alle teorie portate da Malament come controesempi, tentando dunque di riformularle in maniera tale che risultino anch'esse costruite sullo spaziotempo fisico, ed essendo dunque passibili di essere nominalizzate per mezzo del metodo di Field. In questo senso, quello nominalista rimane ancora un programma di ricerca aperto, nella misura in cui ancora è aperta la discussione sulla possibilità di completarlo e invero il tentativo stesso di completarlo (si veda, per un esempio recente di un lavoro in questo spirito, Arntzenius e Dorr (2011) oppure Chen (2017)).

Fintanto che questo tentativo sarà in essere, continuerà a costituire un possibile modo per evitare le conclusioni platoniste dell'argomento di Putnam e Quine, e per potenzialmente invalidare l'argomento stesso.

\section{Conclusioni}

In questo breve contributo, ho cercato di introdurre ad evidenziare alcuni aspetti dell'argomento per l'indispensabilità delle entità matematiche di Putnam e Quine. Questo argomento, che ha dominato la discussione in ontologia della matematica dalla seconda metà del '900 ad oggi, tuttora rimane una delle più valide risorse nelle mani del platonista per difendere la sua posizione, nonostante esistano risposte e critiche di stampo nominalista all'argomento, prima fra tutte quella di Field, volte a disinnescare questo marchingegno e permettere di combinare nominalismo e realismo scientifico a dispetto di quello che Putnam e Quine volevano dimostrare, per quanto, ad oggi, nessuna di queste sia definitiva.

Nel complesso, l'argomento rimane un vivo punto di discussione, capace ancora oggi di sollevare questioni e interrogativi sul significato dell'applicabilità della matematica nelle teorie scientifiche, e sulle sue implicazioni.

\section{RINGRAZIAMENTI}

Vorrei prima di tutto ringraziare gli organizzatori del convegno "Lost in Physics and Metaphysics" per una giornata di studi estrema- 
mente proficua e stimolante, e gli altri partecipanti al convegno per i loro interventi ricchi di spunti interessanti. Vorrei inoltre ringraziare Cristian Mariani per alcuni utilissimi suggerimenti su una versione precedente di questo contributo. Qualunque errore rimasto è mia sola responsabilità.

\section{BIBLIOGRAFIA}

Arntzenius F. and Dorr C. "Calculus as geometry", Chapter 8 of Space, Time, and Stuff, Arntzenius, F., pp. 213-270, Oxford University Press, Oxford, 2011.

Chen E.K. "An intrinsic theory of quantum mechanics: Progress in Field's nominalistic program, part I”, Preprint, 2017. URL $=<\mathrm{http}: / /$ philsci-archive.pitt.edu/ 13083/> (Consultato il 01/04/2018)

Colyvan M. "Indispensability Arguments in the Philosophy of Mathematics", The Stanford Encyclopedia of Philosophy (Spring 2015 Edition), Edward N. Zalta (ed.), URL $=<$ https://plato.stanford.edu/archives/spr2015/entries/ mathphilindis/>. (Consultato il 01/04/2018)

Field H.H. Science Without Numbers: A Defence of Nominalism, Blackwell, Oxford, 1980.

Kitcher P. The Nature of Mathematical Knowledge, Oxford University Press, New York, 1984.

Malament D. "Science without Numbers by Hartry H. Field", Journal of Philosophy, 1982, 79 (9):523-534.

Putnam H. Mathematics, Matter and Method, Cambridge University Press, Cambridge, 1975.

Putnam H. "Philosophy of Logic", reprinted in Mathematics Matter and Method: Philosophical Papers, Volume 1, $2^{\text {nd }}$ edition, pp. 323-357, Cambridge University Press, Cambridge, 1979.

Quine W.V. "Two Dogmas of Empiricism", reprinted in From a Logical Point of View, $2^{\text {nd }}$ edition, pp. 20-46, Harvard University Press, Cambridge, MA, 1980.

Quine W.V. "Five Milestones of Empiricism", in Theories and Things, pp. 67-72, Harvard University Press, Cambridge, MA, 1981.

Quine W.V. "Reply to Charles Parsons", in The Philosophy of W.V. Quine, L. Hahn and P. Schilpp (eds.), pp. 396-403, La Salle, ILL: Open Court, 1986.

Wigner E. "The unreasonable effectiveness of mathematics in the natural sciences" Communications in Pure and Applied Mathematics, 1960, 13:1-14. 